\title{
NOTAS SOBRE LA BRIOFLORA BALEAR. 4
}

\author{
J.A. ROSELLO
}

RESUMEN: Se citan cuatro briófitos nuevos para la flora balear: Isotheciu alopecuroides (Dubois) Isov., Neckera complanata Hüb., Phascum cuspidatum Hedw. var. piliferum (Hedw.) Hook. E Tayl., Tima bavarica Hessl. Se señalan nueve especies que constituyen novedad para la flora de la isla de Menorca. Se excluye el Plagiomnium cuspidatun (Hedw.) T. Kop. del catálogo florístico balear.

SUMMARY: The following bryophytes are quoted for the first time in the bryological flora of the Balearic Islands: Isothecium alopecuroides (Dubois) Isov., Neckera complanata Hüb., Phascum cuspidatu Hedw. var. piliferun (Hedw.) Hook. E Tayl., Timnia bavarica Hessl. Nine species are new for the flora of Menorca. Plagiomium cuspidatum (Hedw.) T. Kop. is excluded from the flora of the Balearic Islands.

\section{INTRODUCCION}

A pesar de los ya numerosos trabajos publicados sobre la brioflora de Baleares el catálogo florístico dista mucho aún de quedar completo. Desde la aparición del último catálogo briológico balear (Vives, 1976) se ha incrementado en más de treinta táxones las novedades corológicas a nivel de archipiélago, lo que representa un aumento del $12 \%$ respecto al catálogo mencionado.

No obstante el conocimiento briológico de las diferentes islas es muy desigual ya que la tradición briológica en las Baleares orientales (Mallorca y Menorca) no es comparable a los escasos y fragmentarios datos de que disponemos sobre las restantes islas.

Como contribución a un mejor conocimiento corológico de la brioflora balear, presentamos los resultados de las últimas prospecciones florísticas realizadas en Mallorca y Menorca. En Mallorca hemos centrado las prospecciones en el estudio de localidades situadas en la zona montañosa de la isla, ya que disponemos de muy pocos datos relativos a la flora y vegetación briológica de la Sierra de Tramuntana. En Menorca hemos visitado, preferentemente, aquellas localidades situadas sobre sustrato ácido, no sobrepasando una altitud superior a los $150 \mathrm{msm}$.

La nomenclatura de hepáticas y musgos, que están ordenados alfabéticamente, sigue a Duell (1983) y a Corley et al. (1981) respectivamente, con la excepción de Zygodon rupestris, para el que seguimos la propuesta de Karttunen (1984).

Las novedades florísticas para Baleares van seguidas por dos 
asteriscos y las que constituyen novedad insular de uno.

Los pliegos testimonio de las especies señaladas se encuentran en el herbario particular del autor.

\section{RESULTADOS}

\section{Hepaticae}

Cololejeunea rossettiana (Massal.) Schiffn. (*)

Menorca: Barranc d'Aljandar (EE82); epífita sobre un tocón de Ficus carica, junto a Radula sp. Estéril.

Corsinia coriandrina (Spreng.) Lindb.

Menorca: citada por Knoche (1921) en los alrededores del Barranc d'Aljandar, no se había vuelto a encontrar. La hemos recolectado estéril, formando extensos céspedes en taludes arcillosos en Favaritx (EEO2), Mongofre Nou (EE02) y en Sa Bassa Verda d'Algaierencs (EE73); sobre sustrato ácido.

Frullania tamarisci (L.) Dum. (*)

Menorca: abundante sobre las rocas silíceas de Llinaritx Nou (EE92), Favaritx (EE02), y en los bajos de la Enclusa (EE82), junto a Grimmia trichophylla. Estéril.

Pellia fabbroniana Raddi

Menorca: Ses Canassies (EE91), abundante en las acequias. En esta isla sólo era conocida del Barranc d'Aljandar (EE82) (Rodríguez, 1875). Estéril.

Petalophyllum ralfsii (Wils.) Nees \& Gottsche (*)

Menorca: rara en un prado en el barranco de Llucalari (EE91) con Riccia lamellosa y Fossombronia sp.; Cala En Porter (EE91), en los márgenes del camino. Estéril.

Scapania aspera Bernet

Mallorca: abundante en la zona montañosa de la isla: Gorg Blau (DE80), Escorca (DE80), Massanella (DE90), Puig Major (DE80), Tomir (DE90), Mola de Planicie (DE59). Estéril. Citada únicamente en Sóller (Nicholson, 1907).

Riella helicophylla (Bory \& Mont.) Mont. (*)

Menorca: torrente de Favaritx (FE82), cerca del mar; charcas temporales de agua en el Port d'Addaya (FE02); Barranc d'Alfandar (EE82) y entre Cala Pregonda y Binimel.là (EE83). En Baleares sólo era conocida de Ibiza (Cros, 1982).

\section{Musci}

Amblistegium riparium (Hedw.) B.S.G. (*)

Menorca: abundante en la Bassa Verda d'Algairencs (EE73), en aguas estancadas. Estéril.

Bryum argenteum Hedw. (*)

Menorca: sobre tierra en el Poblat d'En Galmés (EE91), junto a Funaria hygrometrica. Estéril. 
Cratoneuron commutatum (Hedw.) G. Roth

Mallorca: en acequias, fuentes y torrentes: Cala Banyalbufar (DD59), Massanella (DE90), Escorca (DE80), Estellencs (DD58) y Fornalutx (DE70). Estéril. Conocida anteriormente de Miramar (Hermann, 1914) y Deià (Koppe, 1965).

Dicranum scoparium Hedw.

Mallorca: bajo los arbustos de Hypericum balearicum en el Puig Major (DE80), Tomir (DE90), Serra dels Teixos (DE80) y Puig Roig (DE81), a altitudes superiores a los $800 \mathrm{~m}$. Estéril.

Distichium capillaceum (Hedw.) B.S.G.

Mallorca: Massanella (DE90), en las grietas de los peñascos orientados al norte, a $1.250 \mathrm{~m}$. Con esporófitos. Conocida previamente de los alrededores de Sóller (Hermann, 1914).

Encalypta streptocarpa Hedw.

Mallorca: Mola de Planicie (DE59), Puig Major (DE80), Tomir (DE90), en los taludes. Con propágulos. Citada previamente en Massanella (Sloover, 1967).

Grimmia trichophylla Grev.

Menorca: Llinaritx Nou (EE92) y Favaritx (FE02), sobre los esquistos. Estéril. En Menorca se conocía únicamente de Ferreries (Rodríguez, 1875).

Isothecium alopecuroides (Dubois) Isov. ( $\star \star)$

Mallorca: raro en el Puig Major (DE80), en las grietas de las rocas y bajo Hypericum balearicum. Estéril.

Leptodon smithii (Hedw.) Web. \& Mohr. (*)

Menorca: Mongofre Nou (FEO2), sobre las areniscas y junto a Radula sp. Estéril.

Neckera complanata Hüb. ( $* *)$

Mallorca: Puig Major (DE80), en las grietas de rocas calizas resguardadas, así como en las paredes verticales de un torrente.

Orthotrichum speciosum Nees

Mallorca: Puig de Massanella (DE90), epifito sobre Rosmarinus officinalis, raro. Con esporófitos. Citado con anterioridad en el Puig Major (Nicholson, 1907).

Phascum cuspidatum Hedw. var. piliferum (Hedw.) Web. \& Mohr. ( $\left.* \star)^{\prime}\right)$ Menorca: sobre la arcilla en el camino del Barranc d'Aljandar en Cala Galdana (EE82). Con esporófitos. La var. cuspidatum ya era conocida en Mallorca y Menorca.

Plagiomnium cuspidatum (Hedw.) T. Kop.

La presencia de P. cuspidatum en Baleares está fundada en la cita de Sloover (1967) basada en ejemplares recogidos en Massane$1 l a$ y Escorca. La indicación de Koponen (1971, 1980) de que formas reducidas de $P$. undulatum habian sido confundidas con otras especies del género, en la región mediterránea, nos llevó a considerar si P. cuspidatum formaba, efectivamente, parte de la brioflora balear o, si por el contrario, se había confundido con P. undulatum, especie muy abundante en las localidades señaladas por Sloover. El estudio de testimonios recogidos por Sloover (depositados en BCB) indica que pertenecen a P. undulatum estéril, con abundantes estolones. Cabe excluir a P. cuspidatum del catálogo florístico balear. 
Polytrichum juniperinum Hedw.

Mallorca: rellanos arcillosos en el Puig Caragoler (DE91) y en Es Castellots y Cases de Neu des Galileu (DE80). Estéril. Conocida únicamente de Massanella (Sloover, 1967).

Timmia bavarica Hessl. (**)

Mallorca: en las grietas de las rocas en el Puig Major (DE80), entre 1.200 y $1.400 \mathrm{~m}$. Con esporófitos.

Tortula subulata Hedw. (*)

Menorca: Sa Roca de Mercadal (EE92), en los bordes de la carretera. Con esporófitos.

Zygodon rupestris Schimp. ex Lor. (*) propágulos.

Menorca: epífito de Q. ilex en Son Gall (Alaior) (EE92). Con

\section{AGRADECIMIENTOS}

Agradecemos a G. Brassard (Newfoundland) la revisión de Timmia bavarica Hessl. y a Josep R. Gómez la compañía, estímulo y citas que nos ha proporcionado durante las herborizaciones por Menorca.

\section{BIBLIOGRAFIA}

CORLEY, M.F.V. \& AL. -1981- Moses of Europe and the Azores; an annotated list of species, with synonyms from the recent literature. J.Bryol. 11: 609-689.

CROS, R.M. -1982- Algunos briófitos interesantes de las islas Baleares. Acta Bot. Malacitana, 7:141-150.

DUELL, R. -1983- Distribution of the European and Macaronesian liverworts (Hepaticophytina). Bryol. Beitr., 2:1-115.

HERMANN, H. -1914- Ein botanischer Ausflug nach Majorka. Verh. Bot. Ver. Prov. Branderbourg, 54: 239-257.

KARTTUNEN, K. -1984- Zygodon gracilis, Z. rupestris and Z. dentatus, comb. nova (Musci, Orthotrichaceae). History and nomenclature. Ann. Bot. Fennici, 21: 343-348.

KNOCHE, H. -1921- Flora Balearica: étude phytogéographique sur les îles Baléares. Vol. 1. Montpellier.

KOPONEN, T. -1971- A monograph of Plagiomnium sect. Rosulata (Mniaceae). Ann. Bot. Fennici, 8: 305-367.

KOPONEN, T. -1980- A synopsis of Mniaceae (Bryophyta). IV. Taxa in Europe, Macaronesia, NW Africa and the Near East. Ann. Bot. Fennici, 17: 125-162.

KOPPE, F. -1965- Bryologische Beobachtungen auf der Insel Mallorca. Bot. Not., 118: 25-48.

NICHOLSON, W.E. -1907-Contributions to a list of the mosses and hepatics of Majorca. Rev. Bryol., 34: 1-6.

RODRIGUEZ, J.J. -1875- Catálogo de los musgos de las Baleares. Anal. Soc. Esp. Hist. Nat., 4: 41-51.

SLOOVER, J.L. -1967- Quelques bryophytes récueillies à Majorque. Les Naturalistes Belges, 48: 389-394. 
VIVES, J. -1976- Aproximació a la Flora dels Briófits Balears. 1-53 + 10 låm. Barcelona. 